\title{
The problem of counterfactual de re attitudes*
}

\author{
Igor Yanovich \\ MIT
}

\begin{abstract}
The problem of counterfactual attitudes de re was identified by Ninan (2008) as a challenge for standard theories of de re. I show that once counterfactual non-de re attitudes are properly analyzed, trivial composition of such an analysis for them with the analysis of doxastic de re provides the solution to the problem. Thus there is no independent phenomenon of counterfactual attitudes de re, and therefore no problem as such.
\end{abstract}

Keywords: de re, transworld identification, counterfactual attitudes, de credito

\section{Introduction}

An attitude de re is a propositional attitude which can be said to be "about" some object in the actual world. Thus if Janell has a belief which is "about" the actual Rutgers University, she has an attitude de re whose res is Rutgers University. ${ }^{1}$

(1) Janell believes something of the actual object which is Rutgers University. Namely, she believes of that actual object it is in New Jersey. ${ }^{2}$

It is clear that the attitude holder herself is not able to classify her attitude (at least if it is about an empirical individual, as opposed to, say, about a number) as $d e$

* This paper benefitted greatly from discussions at the semantics reading groups at MIT and at NYU. Without the intellectual generosity of Pranav Anand, Gennaro Chierchia, Elizabeth Coppock, Kai von Fintel, Michael Franke, Irene Heim, Salvador Mascarenhas, Dilip Ninan, Paolo Santorio, Robert Stalnaker, Anna Szabolcsi, and the anonymous reviewers for SALT, it would also have been much worse. Though I disagree with Dilip Ninan on the main point of this paper, my work was directly inspired by his. Needless to say, all mistakes there may be are only my responsibility.

1 Within the linguistic literature an altogether different usage of the terms de re and de dicto can often be found (see, e.g., Sharvit 2002 or Keshet 2011), when a linguistic expression $\alpha$ is called de re with respect to a (modal) linguistic expression $\beta$ if the interpretation of $\alpha$ does not depend on the index introduced by the interpretation of $\beta$, and de dicto if it does. On the other hand, in logic one can encounter yet another usage of the terms, where a formula is called de re if it involves quantification into a modal context. E.g., $\forall x \square \phi(x)$ is de re in that sense, while $\phi(x)$ and $\square \forall x \phi x$ are de dicto. These two pairs of technical notions are, so to speak, genetically related to, but very different conceptually from the notions of $d e$ re and de dicto used in this paper.

2 In the examples, I use a sans serif font to denote pure, non-linguistic content. I use normal font to represent linguistic expressions. 
$r e$, for she normally would not be able to tell with certainty if the attitude is about some real object. Of course, the holder of a potentially de re attitude should assume it is, but she may be wrong.

Thus the characterization of an attitude as de re is always external to the bearer of the attitude. In the oversimplified case, it is the actual world as such which determines whether an attitude is de re. In more realistic cases, some observer assumes the authority of declaring (some of) certain attitudes to be de re.

So whenever we say an attitude is de re, what we mean is that some authority external to the attitude holder has declared that that attitude is about some actual object. What exactly must hold for the external authority to make such a declaration is an important question, and the answers to it create different theories of de re.

Turning to attitude reports $d e$ re, such a report is a linguistic expression which ascribes to an agent (or agents) an attitude de re. Linguistic expressions as such, at least in languages like English, cannot be marked explicitly as attitude reports de $r e$, so the relevant reading of the respective expression is always one of the several readings available for it. In particular, the DP Rutgers University in 2 can be read as de re, in which case the sentence roughly means 1 ; or as de dicto, in which case Janell herself can describe her belief as I believe that Rutgers University is in NJ, but no connection between the content of belief and the real world is necessary. In usual conversational practice, both readings of a sentence like 2 are often true, and moreover, both may be intended to be conveyed, but there are also uses which can only be made sense of on only one of the readings.

\section{(2) Janell believes that Rutgers University is in New Jersey.}

The de re reading of 2 may be true even if Janell herself does not know the very name 'Rutgers University'. If the big question on the attitude content side is what must hold in order for the external authority to declare a certain attitude to be de re, the big question on the side of attitude reports is how a DP which could not be used by the attitude holder herself may nevertheless be used when reporting her attitude.

This paper does not aim to settle these big questions. Its main concern is not the general theory of de re attitudes, but the special theory of counterfactual de re attitudes. While the general theory of de re is usually tacitly assumed to apply to all kinds of attitudes, the cases actually discussed and analyzed are for the most part cases of belief and knowledge de re. But it turns out that extending the usual theories to different kinds of attitudes may be not as straightforward as one might wish. In particular, counterfactual de re attitudes were argued by Ninan (2008), Ninan (2010) to pose a challenge to the Kaplan-Lewis-style general theories of de re. I counter Ninan's position by arguing that while we do indeed need to think carefully about what happens in counterfactual de re (just as Ninan suggests), a proper analysis shows that such attitudes do not actually pose a threat to the Kaplan- 
Lewis-style theories, nor do they lend them any additional support. The special theory of counterfactual de re I am going to propose may be implemented within different general theories of de re attitudes and de re attitude reports.

The plan of the paper is as follows. In Section 2, I sketch a specific Kaplanian theory of $d e$ re, in order to have a specific theory to work with. I do find the version I put forward reasonable, and stand by my choices, but I do not defend it as the best possible theory of de re - there are more things to it than it can be discussed in this paper. In Section 3, I present Ninan's puzzle of counterfactual de re, and explain why it can be viewed as a threat to Kaplanian theories such as the one presented in Section 2. Then in Section 4, I step back a little and analyze from scratch the modes of counterfactual attitudes without a de re component, distinguishing purely de dicto and de credito modes. In Section 5, I return to counterfactual de re, and show that it essentially is doxastic de re (which can be analyzed along the Kaplan-Lewis lines, or otherwise) composed with the de credito mode of the counterfactual attitude, so there is no independent phenomenon of counterfactual de re. This point does not depend on the particular choice of theories for de re and de credito.

\section{The sketch of a Kaplanian theory of (doxastic) de re}

I will now present the sketch of a Kaplanian theory of (doxastic) de re. Framing it from the start as a theory of only doxastic de re involves a certain amount of look-ahead, but it is not unjust given that actual theories I will be heavily using to build this sketch were in practice designed to deal with, and tested on, doxastic cases.

The theory will have three layers: 1) an informal theory of de re attitudes, 2) its formalization within the centered worlds framework, and 3) a semantics for linguistic attitude reports based on the formalization. Why not go to the most specific formulation of the theory first, skipping the informal part? The formal parts have to use the concepts introduced informally, such as the genetic character of a name. Thus the informal characterization is actually the main part of the theory. The informal part of the sketch below sticks fairly closely to Kaplan (1969) (or at least to how I read him), with splashes of insights from Lewis 1979 every now and then. I formalize the informal story within the Lewisian theory of genuinely first-person knowledge; the linguistic wrapping for the analysis is done in a way which to my knowledge has not been used before for such purposes, and involves making the interpretation rules of the translation logic to do the job. Obviously those choices are not the only ones possible, though I stand by them at the moment. ${ }^{3}$ But if you

3 As by reasonable choices for a descriptivist theory of de re; at this point, I have no opinion as to whether the descriptivist perspective is the right one, though I believe that certain insights descriptivist investigations have brought about should survive in any reasonable theory of de re. 
The problem of counterfactual de re attitudes

disagree, this theory can still serve as a representative for the family of Kaplanian approaches to de re, which Ninan takes his puzzle to argue against.

\subsection{Informal theory of (doxastic) de re}

Kaplan's take on de re with respect to "empirical individuals" (as opposed to abstract entities like numbers, which I will have to ignore in this paper) is this: $j$ 's doxastic attitude $\phi$ is de re about individual $a$ iff there is a description $\alpha$ such that 1) $\alpha$ denotes $a$ in the actual world, 2) the genetic character of how $j$ actually acquired $\alpha$ involves the actual $a, 3) \alpha$ is a vivid name, namely, it a) is sufficiently rich in detail (vivid), and b) represents to $j$ a character in $j$ 's inner story, and 4) the belief $\phi$ involves $\alpha$. We will now elaborate on all parts of this analysis.

There are two parts of this analysis of $d e$ re: one concerning the internal workings in the mind of the attitude holder (conditions 3a, 3b), and another concerning the connection between those internal workings and the actual world (conditions 1, 2).

We start with the attitude holder's mind. Using Kaplan's metaphor, she has a sort of an inner story in her head: she has some idea about who the people and things in the world she lives in are, and what relations with each other they are in. Some part of that inner story may be very abstract knowledge: e.g., she might know that giraffes exist, without ever having been en rapport with any particular giraffe, or caring about any. But there are also specific characters in the inner story: the attitude holder usually believes in the existence of certain individuals. We can call those characters of the story her ideas of individuals. ${ }^{4}$

Kaplan calls the inner representation of those ideas of individuals vivid names. There are two aspects of the concept which are amalgamated in Kaplan 1969, but which I wish to disentangle. First, names as such are literally equal to their descriptive content. Adopting a Lewisian perspective, we can distinguish the truly impersonal part of the descriptive content (including properties such as 'has gray hair') and the first-person part (' $x$ is such that I attribute to $x$ the property of being seen by me, in the de se way, yesterday in the cafe'). But even if a particular name is such that the attitude holder believes describes one individual in each of her doxastic alternatives, it is not yet certain that she really uses the name to represent a single individual. E.g., Kaplan states (cf. p. 204 of op. cit.) that merely knowing some proper name does not create by itself an inner character of the attitude holder's story. Whether or not she considers some name to represent a character for her is not a neutral, impersonal fact.

What does it mean for a description to represent a single individual for the

4 Cf. conceptual individuals of Asher 1987, formally anchored discourse referents of Kamp 1990, ideas of Edelberg 1992, internal anchors of Kamp, Genabith \& Reyle 2011, to name just a few, which share the intuition, though not the implementation. 
attitude holder? Take a description like 'the youngest spy'. If Janell believes there are spies at all, there would usually be exactly one person in each doxastic alternative of Janell's who satisfies the description. But that does not mean such a person is an inner character for Janell. Whether some description $\alpha$ is considered by the attitude holder to be the description of some particular, specific person can be probed using questions of the form "Do you know who $\alpha$ is?" Two possible readings of 3 may help to clarify it:

(3) Janell: The President of Rutgers must have graduated from it.

On one reading, Janell does not know much about who the President might be, but Holmes has convinced her using his deductive method that it just cannot be that the president graduated from a different university. If Janell had that reading in mind when uttering 3, then when we ask her whether she knows who the President of Rutgers is, she won't be able to say anything beyond the fact that, well, it is the President of Rutgers. The description simply specifies the denotation of the name.

On the other reading, Janell might not know who exactly the President is: she might not know the President's name, what he or she looks like, what academic field she or he specialized in, etc. etc. The most detailed description of the President she has may be exactly the same as in the previous case. But now she, unlike in the first scenario, thinks about the President in a specific way, as of a particular person. (For instance, she might be really interested in what the President is like because she is preparing to petition him or her, and thus she is gathering all the information she can to increase the chances for success.) When we ask her who the President of Rutgers is, in this situation she will be able to answer at least something like "I don't know much about him or her at the moment, but I really need to know what sort of person they are, and I hope I will soon find out." In this case, the President of Rutgers is a legitimate inner character - Janell thinks about her or him as of a particular person, not just as of whoever a certain definite description actually refers to. The description may be the same in both cases, but the way Janell treats it is different.

Kaplan uses a picture metaphor to explain his theory of names. It may be instructive to elaborate on the way the distinction between being an inner character and being vivid looks like in that metaphor. Janell can look at a picture and judge how detailed the picture is. It may be very vivid, or very vague. At the same time, she can have an opinion as to whether this picture is of some object, or it is just some sort of an artifact, a combination of components of different photographs. It is up to her whether to believe that the picture represents some actual object, and her decision is not determined simply by the level of detail the picture has - though the level of detail may affect her willingness to treat the picture as a representation of something. Similarly with characterhood in general: it depends on the attitude 
holder's choice, and cannot be predicted solely using the descriptive content. ${ }^{5}$

Of course, there is a correlation between the vividness of descriptive content and whether the name represents a particular individual in one's inner story. It is very hard, if not impossible, to use short descriptions like 'the youngest spy' or 'the youngest giraffe' as representing a particular individual. But if someone convinced himself that the youngest giraffe is 4 days old, lives in the Berlin zoo, cannot run yet, etc., it becomes easier for him to start treating the youngest giraffe as a character of the inner story. But there is no level of detail which guarantees that he will. The following example makes this point perhaps most forcefully. Janell logs into some instant messaging service on the internet, with the intention to chat with somebody for a little while. In 15 seconds, she sees a message Hi there!. At this moment, Janell does not know pretty much anything about who sent her that message: she has no idea where they live, what they want, or whether they are wearing shoes at the moment. But she already treats the person "on the other side of the line" as an actual person she is interested in. It is already an inner character of hers.

So on the internal side, a description must meet two criteria for being a vivid name: it should be sufficiently detailed (vivid), and it should represent a particular inner character for the attitude holder. Having such a vivid name is a pre-requisite for having a de re attitude. But the attitude holder herself cannot say if her attitude is de re, only the external authority can, depending on the external connections present.

There are two conditions on the external connections of a vivid name in Kaplan's theory (for empirical individuals). First, the vivid name should denote some object, the res, in the actual world. Second, the genetic character of how the name was acquired by the attitude holder must have a causal connection with the actual res.

Using Kaplan's picture explanation, a picture may be a picture of something: the object which was photographed. The picture may resemble what it is of, or it may not, but that does not change the genetic character of the picture (that is, how it came to be). A picture may also have absolutely nothing it is directly of in the actual world - for instance, if it is a mechanical combination of other images.

Similarly for the genetic character of descriptions in general: Janell may have acquired a belief about the actual youngest giraffe because she went to a zoo, and was told there all sorts of details about it. In this case, her beliefs are causally connected to the actual giraffe in the zoo, even though they may be wrong. In the case when her beliefs are wrong, her name is of that actual giraffe, but does not denote it. On the other hand, Janell may have been completely delusional, and may have invented without any rapport with reality a whole story about the youngest

5 Technically, one can view that as a part of descriptive content, if one allows direct self-ascription of treating a certain name as an inner character. I steered clear of this option simply to avoid mixing self-ascription of relations to transworld entities and in-world entities. Keeping the two separate makes it easier for me to think clearly about them, at least at the moment. But this is a matter of taste. 
giraffe which she now believes is true. Yet accidentally it so happens that the story is absolutely true. In that case, her description of the youngest giraffe will denote the actual giraffe, but will not be a description of him.

It is important to elaborate here on Lewis's disagreement with Kaplan, noted in footnote 14 of Lewis 1979: 2. Lewis does not impose an independent condition concerning genetic character on de re. Instead, he only requires that the first-person beliefs of the attitude holder connecting her to an idea of individual were in fact actually true. If the attitude holder believes that she has seen the youngest giraffe yesterday, and she has indeed seen the actual youngest giraffe yesterday, her belief is true. But there is no further requirement on genetic connection between the description and the res. We now consider a case where there is a difference between the two views.

Suppose Janell have once met Mayor Ortcutt, and thus came to know a handful of things about him (e.g., that she is taller than him, that he likes to boast about his achievements as a mayor, etc. etc.) She still believes all those things, and in fact has a very vivid description of Ortcutt which selects him in the actual world, but she has completely forgotten that she came to know all that from meeting Ortcutt in person. She often thinks about Ortcutt, and he is clearly an inner character of hers.

Now set that scenario aside, and consider the following slight variation. Janell's beliefs are just as they were in the previous scenario. But she have never met Ortcutt. Accidentally, all she believes about him is true, but the actual Ortcutt had nothing to do with it. There was no actual connection between the real Ortcutt and how Janell came to believe all those things about the character of her inner story which shares a lot of properties with the actual Ortcutt.

I find it reasonable to say that in the first case, Janell has a de re belief about Ortcutt, and that in the second case, she does not. But as Janell's beliefs are the same in two scenarios, Lewis cannot distinguish between the two. Only adding the genetic character into the mix allows us to do that. ${ }^{6}$

But while Lewis requires less actual connections between the attitude holder's building of the description and the res, at the same time he requires more than Kaplan does when he says that the vivid name must necessarily be a relation of acquaintance. Where Kaplan only requires the genetic character to connect the actual attitude holder to the actual res, Lewis requires for the attitude holder to

6 Lewis, if I have him right, disagrees we need to do that, so it is not that I have proven him wrong. Cf. Lewis 1983: 11, where Lewis discusses the case of the delusional detective Scholmes, who has a very vivid idea of the murderer which does not correspond to "anyone real". Even in this case when the relation of acquaintance does not select anybody in the actual world (in addition to there being no genetic link between anyone real and Scholmes's idea of individual), Lewis is prepared to allow existential generalization and say that there is someone whom Scholmes believes to be the murderer (though I still do not think Lewis would call this a case of de re belief, for there is no actual res). 
self-ascribe first-person acquaintance with the counterparts of the res. In this respect, I do not disagree with Lewis, but my implementation is different from his. His requirement of direct self-ascribed involvement I replace with a requirement on the vivid name to represent a particular individual. Treating a description like an inner character creates exactly the kind of first-person connection between the description and the attitude holder which is intuitively needed (cf. fn. 5 above).

The accidental de re case shows the denotation and the genetic character requirements are independent, and that the vivid name and the genetic character requirements are independent as well. What about the denotation requirement and the vividness-of-content requirement?

Obviously there is an interconnection between the descriptive content and the actual denotation of a name: if the name is not vivid enough, it can hardly denote a single object in the actual world. So it might seem that the vividness requirement is superficial. If I have Kaplan right, he considers the vividness requirement to do an independent job here as well, and this position may be argued for using the Holmes-and-murderer example. Immediately upon learning of the crime, Holmes forms a very vague idea of individual which he can only describe as "the murderer (of such and such)" at the moment. The description, however vague, will still select the right person in the actual world (assuming that there was only one murderer of such and such). Yet we would normally be unwilling to say that there exists someone who Holmes believes is the murderer. Kaplan explains this unwillingness by the lack of detail in the description. So the two requirements are indeed independent.

Before we move on to our formalization, there is an important remark to make on the overall status of Kaplan's conditions for de re. Kaplan's original concern is epistemological in nature. He is interested in the conditions which should obtain in order for the external authority to declare exportation (or trans-world identification, or substitution) valid. But the conditions as formulated are just a normative ideal. In most real-life cases involving "empirical" individuals, we will never have such a perfect match as they require. So rather than finding exportation perfectly valid, the external authority would most of the time rather find it simply reasonably acceptable. ${ }^{7}$ It depends on the particular interests of the external authority which conditions to relax, and by how much. For instance, in the Holmes-and-murderer example, the authority may have absolute faith in Holmes, and thus completely relax the vividness requirement. Such an authority will find the claim that there is someone who Holmes believes to be the murderer quite acceptable. Similarly, Anand 2006: 20 reports that he finds valid substitution of 'the shortest spy' with the name of the actual shortest spy in a belief report even when the believer only has the description 'the shortest spy' for that person, which is not a vivid name for her.

7 Cf. Kaplan 1969: 203, where it is explicitly discussed how to vivisect a name $\alpha$ in order to turn it into some $\alpha^{\prime}$ which would still allow us to perform a (relatively sensible) exportation. 
Whether one finds valid such substitution or not depends on what standards one sets: if one is willing to identify somebody's beliefs not involving an inner character as beliefs about an actual individual, so be it. But whether you are willing to accept a certain case of relaxation or not, it is a fact that if we were to strictly adhere to the normative ideal, we would end up not declaring any attitudes to be de re about empirical individuals. Some level of relaxation is always necessary in real-life cases.

\subsection{Formalization of our Kaplanian theory}

For the formalization, we use Lewis's centered world theory of representation for belief. We will use a meta-language $L$ interpreted in a first-order (FO) Kripke model augmented by some machinery needed to accommodate our analysis of de re.

A “de re-ready" FO Kripke model $\mathfrak{M}$ for language $L$ is a tuple of the form $\left\langle W, R,\{D(w)\}_{w \in W},\{V(w)\}_{w \in W}\right.$, chrc, vivid, gntc $\rangle$. The first four parts are similar to the standard Kripke model for FO modal logic, the only difference being in that we have a Lewisian take on accessibility. Thus $W$ is the set of worlds; $R$ is the doxastic centered world accessibility relations (in type $D \times W \rightarrow D \times W$, and restricted by a postulate that a pair $\langle a, w\rangle$ cannot make any relation true unless $a \in D(w)$ ); each $D(w)$ is the domain of individuals living in world $w$; and $V(w)$ is the valuation function providing the predicate and function symbols with extensions in each world. We call $D$ the union of all $D(w)$ with $w \in W$.

$\operatorname{Dox}(j)$ at world $w$ is the abbreviation for $\left\{\left\langle x, w^{\prime}\right\rangle \mid\langle j, w\rangle R\left\langle x, w^{\prime}\right\rangle\right\}$, that is, the set of centered worlds doxastically accessible for $j$ in $w$. For each $\operatorname{Dox}(j)$ at $w$, we also define the set of worlds figuring in it: $U_{D o x(j)}:=\left\{w^{\prime} \mid \exists x:\langle j, w\rangle R\left\langle x, w^{\prime}\right\rangle\right\}$.

We define selection functions on $\operatorname{Dox}(j)$ sets. A function $f: D \times W \rightarrow \operatorname{Pow}(D)$ is a selection function on $\operatorname{Dox}(j)$ if the following is true: 1) $\forall\langle x, w\rangle \in D \times W$ : $\forall x \in f(\langle x, w\rangle): x \in D(w)$; and 2) $\forall\left\langle x, w^{\prime}\right\rangle \in \operatorname{Dox}(j): \forall y, z:\left[y \in f\left(\left\langle x, w^{\prime}\right\rangle\right) \wedge z \in\right.$ $\left.f\left(\left\langle x, w^{\prime}\right\rangle\right)\right] \rightarrow y=z$. So a selection function receives centered worlds and returns sets of individuals living in them; moreover, if it is on $\operatorname{Dox}(j)$, then for any centered world in $\operatorname{Dox}(j)$ it returns a single individual. Selection functions on sets of doxastic alternatives are basically the instrument of unique identification across those alternatives, and are the formal equivalent of names of the informal theory above. (But not of vivid names, which need to satisfy further constraints.)

For a selection function $f$ on $\operatorname{Dox}(j)$, we define the descriptive content $D C_{\operatorname{Dox}(j)}(f)$ as the set of all properties holding of the selected individual and the world's center: $\left\{\phi \mid \forall\left\langle x, w^{\prime}\right\rangle: \phi(x)\left(\imath f\left(\left\langle x, w^{\prime}\right\rangle\right)\right)\left(w^{\prime}\right) \wedge \exists y: \neg \phi(x)(y)\left(w^{\prime}\right)\right\}$. Such properties include both genuinely relational properties, which represent the first-person-way connections between the attitude holder and the name, and properties where the center argument plays no role, e.g., $\lambda x \lambda y$.red $(y)$, which represent the impersonal, objective characteristics true of the objects selected by $f$ in all doxastic alternatives, but not 
properties which do not depend on the non-center argument, to avoid including in the descriptive content of $f$ the genuinely non-relational properties which the attitude holder ascribes to herself.

We can rank selection $f$-s on same $\operatorname{Dox}(j)$ by their descriptive content, so that whenever $D C_{\operatorname{Dox}(j)}\left(f_{1}\right) \subset D C_{\operatorname{Dox}(j)}\left(f_{2}\right)$, we say that $f_{2}$ is more vivid than $f_{1}$.

So far, we have been in the standard realm of quantified modal logic. But in order to build an account of $d e$ re, we need to go beyond that, for we crucially need to treat the names, or selection functions, as objects which can figure in relations. In the semantics for our metalanguage, we need to allow for quantification over selection functions. Furthermore, we need to have several predicates which express the properties of such functions in relation to individuals.

There are three such predicates. $\operatorname{chrctr}(f)(j)$ is true (in $w)$ if $f$ is a selection function on $\operatorname{Dox}(j)$, and $j$ considers $f$ a character of her inner story. This predicate of the model records each $j$ 's views on who their inner ideas of individuals are.

$\operatorname{vivid}(f)(\operatorname{Dox}(j))$ is true (in $w$ ) if $f$ is a selection function on $\operatorname{Dox}(j)$, and $D C_{D o x(j)}(f)$ is vivid enough. If $g$ is more vivid than $f$, and $f$ is vivid enough, then $g$ is also vivid enough. The extension of this predicate reflects the requirements that the external authority puts onto names in order to consider them vivid enough.

Finally, gntc $(f)(j)(a)$ is true (in $w$ ) if $f$ is a selection function on $\operatorname{Dox}(j)$, and the acquiring of the name $f$ by $j$ was causally connected to the individual $a$. This is a predicate which, again, records the position of the external authority, implicitly present in the model: it is the external authority determines when the threshold for $f$ being crucially causally related to the actual res $a$ for $j$ is passed.

Now we are ready to formulate what objects represent the beliefs of an individual in our formalization, and when such beliefs are $d e$ re.

(4) The beliefs of $j$ (in $w$ ) are given by a pair of objects: the set of centered worlds $\operatorname{Dox}(j)$, and the set of selection functions which represent $j$ 's inner characters $\{f \mid \operatorname{chrc}(f)(j)\}$.

(5) $j$ believes $\phi$ de re about individual $a$ (in $w^{*}$ ) iff there is a selection function $f$ on $U_{D_{\text {ox }}}$ such that:

1) $\quad l\left[D C(f)\left(w^{*}\right)\right]=a \quad$ (the descriptive content of $f$ uniquely identifies $a$ );

2) $\operatorname{gntc}(f)(j)(a) \quad$ (how $j$ acquired $f$ was genetically related to $a$ );

3a) $\operatorname{vivid}(f)(\operatorname{Dox}(j)) \quad\left(D C_{\operatorname{Dox}(j)}(f)\right.$ is vivid enough);

3b) $\operatorname{chrc}(f)(j) \quad$ (name $f$ is an inner character for $j$ );

4) $\forall\left\langle x, w^{\prime}\right\rangle \in \operatorname{Dox}(j)$, it is true that $\phi\left(\imath\left[f\left(\left\langle x, w^{\prime}\right\rangle\right)\right]\right)(x)$. 
Igor Yanovich

\subsection{Linguistic semantics based on our Kaplanian theory}

On the non-technical level, our embedding of the formalization above into linguistic semantics is very straightforward:

(6) If $j$ 's belief $\phi$ is de re about individual $a$, then the sentence ' $j$ believes that $\phi(a)$ ' is true, and it is synonymous with ' $a$ is such that $j$ believes of it $\phi$ ' ${ }^{8}$

Working it out compositionally requires some technical work. The trick is to allow a DP which normally directly denotes $a$ to be read as imposing the conditions in 5. Both with and without exportation, we should get the following meaning:

$$
\begin{aligned}
& \exists f:\left(\imath\left[D C(f)\left(w^{*}\right)\right]=a\right) \wedge \operatorname{gntc}(f)(j)(a) \wedge \operatorname{vivid}(f)(\operatorname{Dox}(j)) \wedge \\
& \wedge \operatorname{chrc}(f)(j) \wedge\left[\forall\left\langle x, w^{\prime}\right\rangle \in \operatorname{Dox}(j): \phi\left(\imath\left[f\left(\left\langle x, w^{\prime}\right\rangle\right)\right]\right)(x)\right]
\end{aligned}
$$

The entry for believe will be straightforward:

$$
\text { 【believe that } \rrbracket_{\langle\langle s,\langle e, t\rangle\rangle,\langle e, t\rangle\rangle}=\lambda p_{\langle s,\langle e, t\rangle\rangle} \cdot \lambda x . \forall\left\langle y, w^{\prime}\right\rangle \in \operatorname{Dox}(x): p(y)(w)
$$

The question is how to get the conditions on $f$ upstairs, and to ensure we have $\imath\left[f\left(\left\langle x, w^{\prime}\right\rangle\right]\right.$ in place of the de re DP in the non-exportation form, and a pronoun bound by it in the exportation form, starting from something like this:

$$
\begin{array}{ll}
\text { a. Without exportation: } & \forall\left\langle y, w^{\prime}\right\rangle \in \operatorname{Dox}(j): \phi(a)(y)\left(w^{\prime}\right) \\
\text { b. With exportation: } & {\left[\lambda z . \forall\left\langle y, w^{\prime}\right\rangle \in \operatorname{Dox}(j): \phi(z)(y)\left(w^{\prime}\right)\right](a)}
\end{array}
$$

It suffices to get from one of those forms to 7. For the other, we can simply supply a method of transforming it into the first form. We will treat the non-exportation form as primary, and derive the exportation form in semantics via usual $\beta$-reduction.

If we only look at the subformula $\phi(a)$ in $9 \mathrm{a}$, it simply does not contain enough information for us to figure out the parts we need to change and add in order to arrive at 7. The de re reading of a DP is sensitive to the linguistic context in which the DP occurs. We need to see the whole formula in 9a before we can transform it into 7. In particular, to build the restrictions on $f$ as in 7, we need to supply $j$ and $\operatorname{Dox}(j)$.

There are several technical options here. We can stipulate an ambiguity in the DP: on one reading, it would denote an individual (or a GQ), on the other, a heavily type-lifted expression taking $\phi$ and believe as arguments. ${ }^{9}$ Or we can stipulate an ambiguity in the verb, as many linguists do, and have a separate de re version of

8 I am intentionally sloppy in not distinguishing an individual and a linguistic expression referring to that individual. No confusion should arise, for the context clearly determines what is meant.

9 Here is what such a meaning can look like, schematically:

$$
\lambda \phi . \lambda A t t . \lambda j . \exists f:\left(\imath\left[D C(f)\left(w^{*}\right)\right]=a\right) \wedge \ldots \wedge A t t\left(\left[\lambda w^{\prime} \lambda y \cdot \phi\left(f\left(\left\langle y, w^{\prime}\right\rangle\right)\right)(y)\left(w^{\prime}\right)\right]\right)(j)
$$


believe plus movement of the res. I chose yet another strategy. It leaves LFs like in 9 intact, so there are no more manipulations in the translation logic (or natural language syntax), but interpretation rules of the translation logic are changed so that the formulas in 9 are true exactly when the meta-logical description in 7 is. ${ }^{10}$ I prefer that option to the approaches more usual in linguistics because it does not force us to stipulate very complex linguistic representations for reasons which have nothing to do with how the actual natural language syntax works.

First, I adopt the assumption that the individual domains $D(w)$ of different possible worlds are disjoint. ${ }^{11}$ Furthermore, I say that the truth value for a formula $\phi(a)$ is defined at $w$ only when $a$ is in $D(w)$. This immediately makes it impossible, in most cases, to interpret formulas like 9 a as we normally do, from bottom up: as the domains of different worlds are disjoint, for any $j$ which is not omniscient there will be some $w \in U_{\operatorname{Dox}(j)}$ where $a$ does not exist.

If we stopped right there, 9a would have been undefined. But we can supply an alternative rule of interpretation, which directly assigns truth conditions to a bigger formula. That may remind one of the idea, described by Kaplan (1969) as Quinian, of treating opaque contexts as special predicates defined on the spot. But we derive those predicates in a very general way, utilizing the meanings of their component parts, and thus we do not need any special justification, different for each particular case. The two non-trivial interpretation rules are these:

$$
\begin{aligned}
& \text { If } a \in D(w) \text {, then: } \mathfrak{M}, w \models P(a) \text { iff } a \in V_{\mathfrak{M}}(P)(w) . \\
& \mathfrak{M}, w \models A t_{D o x}\left(\left[\lambda w^{\prime} \lambda y \cdot \phi(a)(y)\left(w^{\prime}\right)\right]\right)(j) \text { iff } \\
& \text { there is a selection function } f \text { on } \operatorname{Dox}(j) \text { such that: } \\
& \imath\left[D C(f)\left(w^{*}\right)\right]=a \wedge \operatorname{gntc}(f)(j)(a) \wedge \operatorname{vivid}(f)(\operatorname{Dox}(j)) \wedge \operatorname{chrc}(f)(j), \\
& \text { and } \mathfrak{M}, w \models A t t_{D o x}\left(\left[\lambda w^{\prime} \lambda y \cdot \phi\left(\imath\left[f\left(\left\langle y, w^{\prime}\right\rangle\right]\right)\right)(y)\left(w^{\prime}\right)\right]\right)(j)
\end{aligned}
$$

The rule in 10 is standard except for the definedness requirement. It is needed so that when $a \notin D(w)$, it was not just that $\mathfrak{M}, w \not \models P(a)$, for that means $\mathfrak{M}, w \models \neg P(a)$. On the other hand, the rule 11 is new. The idea of the rule is very simple: it simply checks if there is a suitable $f$ satisfying the conditions we stated in 5, and such that substituting $\imath\left[f\left(\left\langle y, w^{\prime}\right\rangle\right)\right]$ for $a$ in the big formula results in a formula which is true in $\mathfrak{M}, w$. Because of how we defined selection functions, it is guaranteed that $\left[f\left(\left\langle y, w^{\prime}\right\rangle\right]\right.$ is singleton, and a subset of $D\left(w^{\prime}\right)$, so $\phi\left(\imath\left[f\left(\left\langle y, w^{\prime}\right\rangle\right]\right)\right)(y)\left(w^{\prime}\right)$ has to be defined, and its truth is checked by the usual bottom-up rules. ${ }^{12}$

10 Cf. Fara 2008 for another system for transworld identification which relies for de re on direct interpretation of simple formulas. The two systems are quite different (because the goals behind them are), but both ultimately use interpretation rules to determine counterparts from other worlds.

11 I conjecture it is not strictly necessary, but a system with overlapping domains would probably be more technically involved, because of potential competition between the two rules.

12 Strictly speaking, this system is not well-defined as stated, because it may in principle allow for substitution with an $f$ formula even when $\phi(a)$ is defined. But the only dangerous case is when $\operatorname{Dox}(j)$ 
Igor Yanovich

We thus have a sketch of how to implement the linguistic analysis for our Kaplanian theory, as informally described in 6, and can finally turn to the problem of counterfactual de re.

\section{Ninan's puzzle of counterfactual de re}

The following scenario is a famous puzzle from Quine 1956 about doxastic de re:

The double vision puzzle:

Janell knows Mayor Ortcutt to be a respectable citizen. One evening, Janell sees a man sneaking around the docks, and thinks: "Uh-oh, that must be a spy". The man Janell sees is in fact Ortcutt, but she does not realize it.

In 12, Janell has two vivid names which are of the same res, Ortcutt. Those names select different individuals in Janell's doxastic alternatives, so there is no contradiction in Janell's de re beliefs that Ortcutt (the mayor) is not a spy, and that Ortcutt (the suspicious man at the docks) is a spy.

So far so good, but in that scenario, Janell may very well have some counterfactual attitudes concerning Ortcutt, under either of the two guises. For example:

Ninan's puzzle:

a. Assume the situation in 12. Furthermore, Janell is imagining the man at the docks she is looking at flying a kite in the Alpes.

b. Assume the situation in 12. Moreover, Janell wishes the man at the docks she is looking at were not at the docks, but in the Alpes flying a kite.

Those counterfactual attitudes are no less about the actual Ortcutt than Janell's beliefs in 12 were. They also support double vision: had Janell imagined Mayor Ortcutt flying a kite in the Alpes, her act of imagination would have been very different from the one described in 13a.

We built above a Kaplanian analysis of doxastic de re. But if we, together with Ninan, apply it literally here, it immediately fails. Here is how Ninan applies it: an attitude is de re if there is a vivid name with the right properties as described in 5. The only difference is that we change the attitude, and with it, the set of (centered) worlds which matters. If so, then the descriptive content of the vivid name in the imagination worlds in 13a includes the property of being in the Alpes, but that descriptive content cannot be true of the actual Ortcutt who is now at the

contains just the evaluation world $w$, that is, when $j$ is omniscient. I do not find it too big of a loss to rule out this case explicitly. And in all other cases, it will be the formulas $\phi\left(\imath\left[f\left(\left\langle y, w^{\prime}\right\rangle\right]\right)\right)(y)\left(w^{\prime}\right)$ which are not defined, so there will never be competition between the two interpretation rules, even though they seem to apply in very similar circumstances. 
The problem of counterfactual de re attitudes

docks. Hence, concludes Ninan, the straightforward Kaplanian account fails for counterfactual de re. ${ }^{13}$

\section{Counterfactual attitudes de credito}

Let us put de re onto the back burner for some time, and consider non-de re counterfactual attitudes. It turns out that such attitudes may have two modes, which I hereby baptize purely de dicto and de credito.

In purely de dicto counterfactual attitudes, the attitude is not "about" any idea of individual of the attitude holder's beliefs. In particular, it can be about an object that she believes does not exist at all:

\section{(14) Purely de dicto attitudes:}

13 Ninan (2008) proposes two solutions for his puzzle, one framed as an extension of the Kaplanian approach, another based on sequenced worlds. Up to a certain point, Ninan makes steps similar to ours, but both of his accounts fail when he tries to do trans-world identification. Explaining why will necessarily involve running ahead and using what we are about to show in the next section.

In the first solution, Ninan proposes that imagination is parasitic on belief (just as we will later). But when there comes a time to determine how we select imaginary versions of Ortcutt's in 13a, he argues that we simply select the same individuals which represent Ortcutt in the belief worlds (he assumes overlapping individual domains). To show it will not do, we go by cases. If the attitude holder can identify individuals across worlds because they are the same individual, she must have access to the individuals' essences. This is implausible. On the other hand, if she cannot identify which individuals in the imaginary worlds correspond to the man at the docks, that is also implausible - she knows who she is imagining flying a kite. Thus the first solution fails because no reliable method of identification is provided. My account in this paper is essentially Ninan's first solution plus a proper analysis of where the method of identification comes from.

In the second solution, Ninan says that the identification of the imaginary counterparts of Ortcutt are made by stipulation. The problem with this solution is that stipulation identifies imaginary kite-flying men with the actual Ortcutt, not with the man at the docks of Janell's beliefs. The only relation between the kite-flying men and the men at the docks is through the actual Ortcutt, not directly. But then Janell herself should not be able to tell she is imagining the man at the docks flying a kite, because she does not have access to how the actual world is. Of course, my account of counterfactual de re, using a "counterpart-selection" function is in a sense an account determining the trans-world correspondents by Lewisian stipulation. But that stipulative identification should be between the doxastic ideas of individuals and their imaginary versions, and be performed in the attitude holder's head, not externally to it. So again, my account is very closely related to Ninan's, but by virtue of making sure the power to make the stipulation belongs to the attitude holder, it successfully finishes what Ninan started.

My criticism of Ninan's accounts, and my solution to his puzzle have both much in common with Anand 2011: Sec. 4. Anand accepts both the stipulative nature of trans-world identity in counterfactuals and its parasitism on beliefs (thus combining the good features of both of Ninan's accounts, just as I do), but he does not analyze de credito or specify where exactly the stipulation comes from, in the manner I do in Section 4 of this paper. Anand and I became aware of each other's work, and of the fact we independently developed similar ideas, during SALT 21. 
Igor Yanovich

Janell believes unicorns do not exist.

a. Janell is imagining a unicorn playing in a meadow.

b. Janell wishes that in some meadow, there played a unicorn.

In contrast to that, counterfactual attitudes de credito are "about some object", that object, or creditum, being one of the attitude holder's ideas of individuals: ${ }^{14}$

\section{De credito attitudes:}

Janell believes France is ruled by a king.

a. Janell is imagining the king of France walking in the garden of Chantilly.

b. Janell wishes the king of France were walking in the garden of Chantilly.

Counterfactual attitudes de credito and purely de dicto are distinguishable for the attitude holder herself: she can tell whether she, for instance, wishes some inner character of hers were happy, or just wishes that there exists a happy person in the world. The distinction is in the attitude holder's head. That is crucially different from the distinction between de dicto and de re doxastic attitudes: both of those look the same to the attitude holder, and only look different to the external authority.

Since de credito and purely de dicto are different even for the attitude holder, the representation of counterfactual attitudes in her head must allow her to make that distinction. Yet if we represent the content of counterfactual attitudes as simple sets of (centered) worlds, the distinction cannot be made. Suppose every alternative of such a set for Janell has a unicorn playing in a meadow. We cannot tell from that whether those unicorns are purely de dicto unicorns (as will necessarily be the case if Janell does not believe in unicorns), or are versions of some particular unicorn Janell has in mind.

We need two further components for the representation of counterfactual attitudes: first, a description of the attitude holder's beliefs; second, a method of identification $g_{i d}$ for the creditum in the counterfactual worlds.

The need for the first of those two is evident: we cannot determine whether a counterfactual attitude is about some believed idea of individual without looking into the beliefs. But what is a method of identification, and why do we need it?

To answer that question, we take from the shelf a miracle of science crafted in the 1960s, Jules Verne-oscope (see Kaplan 1967/1979). This astonishing device

14 The acknowledgement of parasitism on belief for some counterfactual attitudes about individuals is in no way new. See, for instance, Kamp et al. 2011: 351, Maier 2006: 34-35, Ninan 2008: Ch. 2, Anand 2011: Sec. 4, a.o. Along more general lines, the selection of counterfactual worlds is usually done in a two-step fashion by semanticists analyzing counterfactual attitudes like wish: e.g., Heim (1992) selects wishing worlds on the basis of what the attitude holder's doxastic alternatives are. The distinctive feature of my analysis, not having been proposed before to my knowledge, is the treatment of the identification method for the creditum as the part of the attitude's content. 
allows us to see before our own eyes different (centered) worlds in their entirety as if we were looking at a picture or watching a movie - that is, we see all properties there are in the world and where the center is, but we do not see any sort of essences. We give the device to Janell who has an attitude as in 13a, and make the Verneoscope show her a world where there is some guy flying a kite somewhere in the Alpes.

Can she tell whether this centered world is compatible with what she imagined? No, because it is not clear whether he is the correspondent of the man at the docks she is looking at. If he is, then the world is compatible. If he is not, then the world is not. But there is no information in the world as seen through the Verneoscope which would allow us to determine this!

This simply means that the content of Janell's imagination must include something extra besides the two parts we already had: she must have a way to determine who in the counterfactual worlds correspond to her doxastic ideas of individuals. If there were an arrow in the Verneoscope picture pointing to the correspondent of the man at the docks, Janell would have immediately be able to tell whether the shown world is compatible with her imagination. A method of identification adds to worlds arrows of the right sort.

Putting it all together, here is how counterfactual attitudes work, under my proposal. First, there are normal beliefs of the attitude holder: a set of doxastic alternatives plus a set of her ideas of individuals. For each doxastic alternative, there is a set of counterfactual alternatives parasitic on it. ${ }^{15}$ Moreover, there may also be methods of identification which, given an idea of individual and a doxastic alternative, return the correspondent of it in each of the parasitic counterfactual alternatives (if there exists a correspondent). Note that by choosing both a $g_{i d}$ and a set of counterfactual alternatives, the attitude holder effectively not only selects which properties to ascribe to the imaginary correspondent, but also which properties of it to keep constant between her beliefs and her counterfactual attitudes. ${ }^{16}$

15 Why make counterfactual alternatives parasitic on individual doxastic alternatives rather than on the full set? I believe it can be clearly shown using examples of the following form. Let $j$ have a belief about the person she sees at a distance that it is her brother or her father. Let $j$ wish that that person suddenly was beside her, whichever of the two it is, but not the other one of the two (suppose there is some good reason why this is very reasonable). Then whether a world where $j$ 's brother is beside her is compatible with what she wished cannot be determined unless it is determined who $j$ saw. Making counterfactual alternatives parasitic on individual doxastic alternatives solves that.

16 Such methods of identification may seem similar to Lewisian counterpart relations. But there is a crucial difference: at least for Lewis himself, counterpart relations are defined solely in terms of descriptive content (be it impersonal or first-person-way descriptive content), and cannot involve anything else. So if we were to use such a Lewisian counterpart relation for trans-world identification of the creditum, as far as I see, all the arguments about circularity from Lewis 1983 (originally made about the determination of the de se counterpart of the attitude holder) would apply. It is crucial that selection of the correspondents in the counterfactual alternatives does not depend on what holds in those alternatives, and thus no circularity arises. Thus methods of identification essentially are a 
For reasons of space, I cannot give the full formal analysis of de credito along the lines above here, so I will instead give two example treatments: the translation of a linguistic attitude ascription reporting 15a, and its truth conditions in our metalanguage (as opposed to the translation logic for language). We should arrive at such truth conditions by using a recipe similar to the one we used for doxastic de re, only now we are effectively replacing a definite description denoting the doxastic king of France with his imaginary counterparts given by $g_{i d}$. Note that we use as the relevant selection function $\lambda x \lambda v$. $[k i n g . F(\langle x, v\rangle)]$, and do not put on it any of the usual conditions we used for $d e$ re, namely vividness, genetic connection, and existence and uniqueness in the actual world; the only thing we require is that it represent an inner character of the attitude holder. The only new meta-language predicate we introduce specifically for de credito is chose which is true of $g_{i d}, j$, and $f$ iff $j$ chose to use the identification method $g_{i d}$ for the idea $f{ }^{17}$

$$
\begin{aligned}
\forall\left\langle y, w^{\prime}\right\rangle \in \operatorname{Dox}(j): \forall\left\langle z, w^{\prime \prime}\right\rangle: \\
\quad\left(\left\langle y, w^{\prime}\right\rangle R_{\text {cnt }}\left\langle z, w^{\prime \prime}\right\rangle\right) \rightarrow\left(\text { Chantilly }\left(\boldsymbol{l}\left[\operatorname{king}\left(\left\langle y, w^{\prime}\right\rangle\right)\right]\right)\left(\left\langle z, w^{\prime \prime}\right\rangle\right)\right),
\end{aligned}
$$

where $R_{c n t}$ is some counterfactual accessibility relation.

$$
\begin{aligned}
& \exists g_{\text {id }}: \operatorname{chrc}(\lambda x \lambda v \cdot[\operatorname{king}(\langle x, v\rangle)])(j) \wedge \operatorname{chose}\left(g_{i d}\right)(\lambda x \lambda v \cdot[\operatorname{king}(\langle x, v\rangle)])(j) \wedge \\
& \wedge\left[\forall\left\langle y, w^{\prime}\right\rangle \in \operatorname{Dox}(j): \forall\left\langle z, w^{\prime \prime}\right\rangle:\right. \\
& \left.\quad\left(\left\langle y, w^{\prime}\right\rangle R_{c n t}\left\langle z, w^{\prime \prime}\right\rangle\right) \rightarrow\left(\text { Chantilly }\left(g_{i d}\left(\boldsymbol{l}\left[\operatorname{king}\left(\left\langle y, w^{\prime}\right\rangle\right)\right]\right)\left(\left\langle y, w^{\prime}\right\rangle\right)\right)\left(\left\langle z, w^{\prime \prime}\right\rangle\right)\right)\right]
\end{aligned}
$$

\section{Back to counterfactual de re}

As we have established in the previous section, certain counterfactual attitudes are de credito: they put some character of the attitude holder's inner story into counterfactual circumstances.

Now, what about counterfactual de re? In Ninan's puzzle itself, 13, we clearly have a combination of de re and de credito. First we have the actual Ortcutt $o$. Then there is an idea of the man in the docks in the head of Janell, $f_{\text {docks }}$, which happens to be a vivid name of Ortcutt for Janell, and has the right genetic character tied to $o$. Then Janell imagines (if it is 13a) $f_{\text {docks }}$ in different circumstances, namely flying a kite, with some method of identification $g_{i d}$ giving us the correspondents of the man at the docks. (Of course, what actually happens is not that Janell imagines the idea as

device allowing identification by stipulation.

Now, the reader familiar with Ninan's work would have noticed the connection to his account here. The difference between his theory and mine is that he does not explain how, namely in whose head, stipulation happens, and rejects parasitism of counterfactual attitudes on belief, without which proper identification by stipulation is impossible.

17 The actual analyses of specific counterfactual attitudes may be more involved than the simplistic schema given here. For imagination, see, for instance, Higginbotham 2003, Recanati 2007, Stephenson 2011, Anand 2011. 
such: she imagines the counterparts of individuals satisfying the idea in her doxastic alternatives.) The attitude is de re because the counterfactual correspondents are related to the actual Ortcutt by transitivity, through the vivid name of the man at the docks $f_{\text {docks }}$ and the method of identification $g_{i d}$ chosen by Janell.

Can a purely de dicto counterfactual attitude nevertheless be de re? I believe not. Suppose I do not believe unicorns exist, and wish there were a unicorn in my backyard. Accidentally, a weird creature satisfying my quite vague conception of unicorns (perhaps a rhinoceros) is indeed in my backyard. Does it mean that my wish about a unicorn was fulfilled? Not really. I wanted to have in my backyard a mythical creature. This wish is in principle unsatisfiable by any actual creature in the backyard. (Cf. a similar argument in Kamp 1990: 60-61.)

So counterfactual attitudes de re actually involve de re combined with de credito. Once we have an independent story for de re which gives us the representatives of the res in belief worlds, and another story for the counterfactual correspondents in attitudes de credito, we simply plug one into the other:

\section{The "solution" to Ninan's puzzle:}

Counterfactual att. de re $=($ belief $d e r e+$ counterfactual att. de credito $)$

It should be noted that 18 is quite robust: it provides considerable freedom regarding the choice of a theory for belief de re or for counterfactual attitudes de credito. In particular, our Kaplanian account for de re may be replaced with a different account, not necessarily Kaplanian. Similarly, if one has a better story for de credito than the one proposed here, as long as the main points of the story are preserved (namely, that counterfactual attitudes de credito are parasitic on beliefs, and that their content includes an identification method accessible to the attitude holder - the points which I believe should survive in any analysis of de credito), the better story can be plugged into one's story for de re. Of course, the two stories need to have matching sockets, so to speak, but as long as they do, we are fine.

For our analyses from Sections 2 and 4, the composition looks like this:

$$
\begin{aligned}
& \forall\left\langle y, w^{\prime}\right\rangle \in \operatorname{Dox}(j): \forall\left\langle z, w^{\prime \prime}\right\rangle: \\
& \quad\left(\left\langle y, w^{\prime}\right\rangle R_{c n t}\left\langle z, w^{\prime \prime}\right\rangle\right) \rightarrow\left(\text { kite }\left(\imath\left[\text { man.docks }\left(\left\langle y, w^{\prime}\right\rangle\right)\right]\right)\left(\left\langle z, w^{\prime \prime}\right\rangle\right)\right) \\
& \exists f: \imath\left[D C(f)\left(w^{*}\right)\right]=o \wedge \operatorname{gntc}(f)(j)(o) \wedge \operatorname{vivid}(f)(\operatorname{Dox}(j)) \wedge \\
& \wedge \operatorname{chrc}(f)(j) \wedge \exists g_{i d}: \operatorname{chose}\left(g_{i d}\right)(f)(j) \wedge \\
& \wedge\left[\forall\left\langle y, w^{\prime}\right\rangle \in \operatorname{Dox}(j): \forall\left\langle z, w^{\prime \prime}\right\rangle:\left(\left\langle y, w^{\prime}\right\rangle R_{c n t}\left\langle z, w^{\prime \prime}\right\rangle\right) \rightarrow\right. \\
& \left.\rightarrow\left(k i t e\left(g_{i d}\left(\imath\left[f\left(\left\langle y, w^{\prime}\right\rangle\right)\right]\right)\left(\left\langle y, w^{\prime}\right\rangle\right)\right)\left(\left\langle z, w^{\prime \prime}\right\rangle\right)\right)\right]
\end{aligned}
$$

Summing up, Ninan's puzzle turns out to have a trivial solution in the end. Yes, it is wrong to use vivid names selecting actual individuals and genetically related to the actual attitude holder for finding the correspondents of the res in counterfactual 
alternatives. But even when we look at counterfactual attitudes which are not de $r e$, we still have to analyze the de credito mode, where what is being imagined, or wished, etc., is about some object in the attitude holder's mind, so we need to identify the counterfactual correspondents of inner story characters anyway. And when we return to counterfactual de re, we already have a method of identifying "believed individuals" with their counterfactual correspondents. Combining that method with the analysis of belief de re, we get the right results for Ninan's puzzle.

\section{References}

Anand, Pranav. 2006. De de se: MIT dissertation.

Anand, Pranav. 2011. Suppositional projects and subjectivity. Ms., UCSC.

Asher, Nicholas. 1987. A typology for attitude verbs and their anaphoric properties. Linguistics and Philosophy 10(2). 125-197.

Edelberg, Walter. 1992. Intentional identity and the attitudes. Linguistics and Philosophy 15(6). 561-596.

Fara, Delia Graff. 2008. Relative-sameness counterpart theory. The Review of Symbolic Logic 1(2). 167-189.

Heim, Irene. 1992. Presupposition projection and the semantics of attitude verbs. Journal of Semantics 9(3). 183-221.

Higginbotham, James. 2003. Remembering, imagining, and the first person. In Alex Barber (ed.), Epistemology of language, 496-533. Oxford: Oxford University Press.

Kamp, Hans. 1990. Prolegomena to a structural theory of belief and other attitudes. In C. Anthony Anderson \& Joseph Owens (eds.), Propositional attitudes. the role of content in logic, language and mind, vol. 20 CSLI Lecture Notes, 27-90. Stanford: CSLI.

Kamp, Hans, Josef Van Genabith \& Uwe Reyle. 2011. Discourse representation theory. In Dov M. Gabbay \& Franz Guenthner (eds.), Handbook of philosophical logic, 2nd edition, vol. 15, 125-394. Dordrecht: Springer.

Kaplan, David. 1967/1979. Transworld heir lines. In Michael J. Loux (ed.), The possible and the actual, 88-109. Ithaca, NY: Cornell University Press.

Kaplan, David. 1969. Quantifying in. Synthese 19(3-4). 178-214.

Keshet, Ezra. 2011. Split intensionality: a new scope theory of de re and de dicto. Linguistics and Philosophy 33(4). 251-283.

Lewis, David K. 1979. Attitudes de dicto and de se. Philosophical Review 88(4). 513-543.

Lewis, David K. 1983. Individuation by acquaintance and by stipulation. Philosophical Review 92(1). 3-32. 
The problem of counterfactual de re attitudes

Maier, Emar. 2006. Belief in context: Towards a unified semantics of de re and de se attitude reports: Radboud Universiteit Nijmegen dissertation.

Ninan, Dilip. 2008. Imagination, content, and the self: MIT dissertation.

Ninan, Dilip. 2010. Self-location and other-location. Ms., University of St Andrews.

Quine, William Van Orman. 1956. Quantifiers and propositional attitudes. Journal of Philosophy 53(5). 177-187.

Recanati, François. 2007. Imagining de se. Talk at conference Mimesis, Metaphysics, and Make-believe, University of Leeds, June 2007.

Sharvit, Yael. 2002. Embedded questions and 'de dicto' readings. Natural Language Semantics 10(2). 97-123.

Stephenson, Tamina. 2011. Experiential attitudes: Centered situations in the semantics of remember and imagine. Ms., Yale.

Igor Yanovich

MIT Linguistics and Philosophy

77 Massachusetts Avenue, Building 32-D808

Cambridge, MA 02139

yanovich@mit.edu 